\title{
A Discrete choice framework for acceleration and direction change behaviors in walking pedestrians
}

\author{
G. Antonini ${ }^{1}$, M. Bierlaire ${ }^{1}$
}

\begin{abstract}
The walking process is interpreted as a sequence of decisions about where to put the next step. A dynamic and individual-based spatial discretization is used to represent the physical space. A behavioral framework for pedestrian dynamics based on discrete choice models is given. Direction change behaviors and acceleration behaviors are taken into account, both in a constrained and unconstrained formulation. The unconstrained direction changes (keep direction, toward destination) and acceleration (free flow acceleration) behaviors are the same as those introduced in our previous work. In this paper we focus on the definition of the constrained counterparts. A leader follower behavior is interpreted as a constrained acceleration while collision avoidance behavior as a constrained direction change. The spatial correlation structure in the choice set deriving from a simultaneous choice of speed regimes and radial directions is taken into account specifying a cross nested logit model (CNL). Quantitative results are presented, obtained by maximum likelihood estimation on a real data set with more than 10 thousands observed positions, manually tracked from video sequences.
\end{abstract}

\section{Introduction}

Modeling pedestrian behavior is becoming always more important. Several approaches have been adopted by researchers from different fields, such as transportation science, architecture and land use, artificial intelligence and computer graphics, among others. For a detailed review of the state of the art on pedestrian behavior modeling we remind the interested reader to [1].

This paper focuses on the walking behavior of individuals at the operational level, extending the discrete choice set up already defined in our previous work. Here we extend the framework keeping the same spatial discretization and choice set definitions, which are quickly reviewed in the next section. The main contribution of this work is a more detailed analysis of acceleration and change direction models. Inspiration is taken from previous works in transport engineering ([2],[3] among others) and those methodologies are extended and adapted to the pedestrian case. A constrained acceleration model (leader follower) and a constrained direction change model (collision avoidance) are specified and fitted to the DCM framework. Quantitative analysis is performed by maximum likelihood estimation, using real human trajectory datasets, manually tracked from video sequences. The paper is structured as follows. In section 2 we define the modeling elements, in section 3 we describe the behavioral model, in section 4 we present

Signal Processing Institute

Operations Research Group ROSO

Ecole Polytechnique Federale de Lausanne

CH-1015 Lausanne, Switzerland 
the datasets used for the estimation process and in section 5 we present the results. We conclude with final remarks and future works.

\section{Modeling elements}

In our approach the walking process is considered as a sequence of choices on the next step position. The time resolution of such decision making process is of the order of 1 second. Assuming the final destination being exogenous to the model, we are interested in modeling the short range behavior of pedestrians, as a response to their kinematic state and to the presence of other pedestrians. In this context, we define the following modeling elements.

\section{The space model}

A dynamic and individual-based spatial representation is used, which depends on the current speed and direction of the individuals. The adaptive discretization is obtained assuming three speed regimes (accelerated, decelerated and constant current speed) and eleven radial directions, as shown in Figure 1.

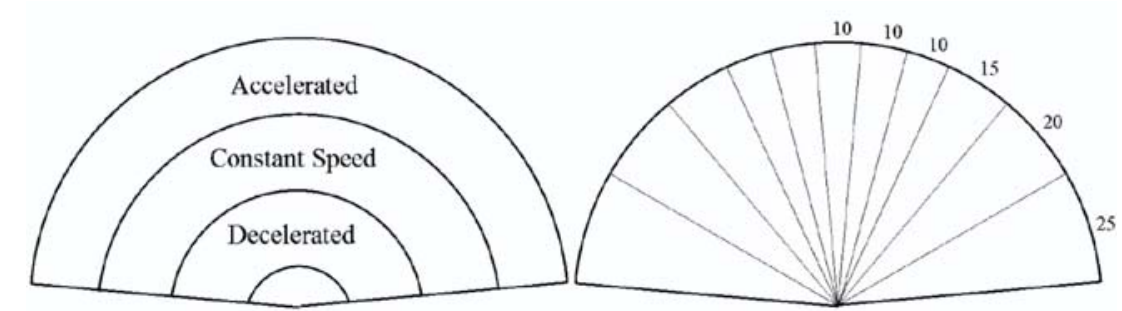

Figure 1: Choice set

\section{Behaviors}

As already said, individuals walk on a 2D plane. Basically, any kind of behavior influencing their movement will result in two kind of observations: changes in directions and changes in speed, i.e. accelerations. In this spirit, we define the general conceptual framework for pedestrian walking behavior, illustrated in Figure 2.

The leafs of the tree show the behavioral patterns we want to capture. In the following, we give a short qualitative description.

» keep direction Pedestrian trajectories in normal conditions ${ }^{1}$ are characterized by a certain regularity. People do not change direction often over time, up to a certain level of density. With the keep direction behavior we identify the tendency of individuals to keep, when is possible, their current direction

${ }^{1}$ With the term normal conditions we mean non-evacuation and non-panic situations. 


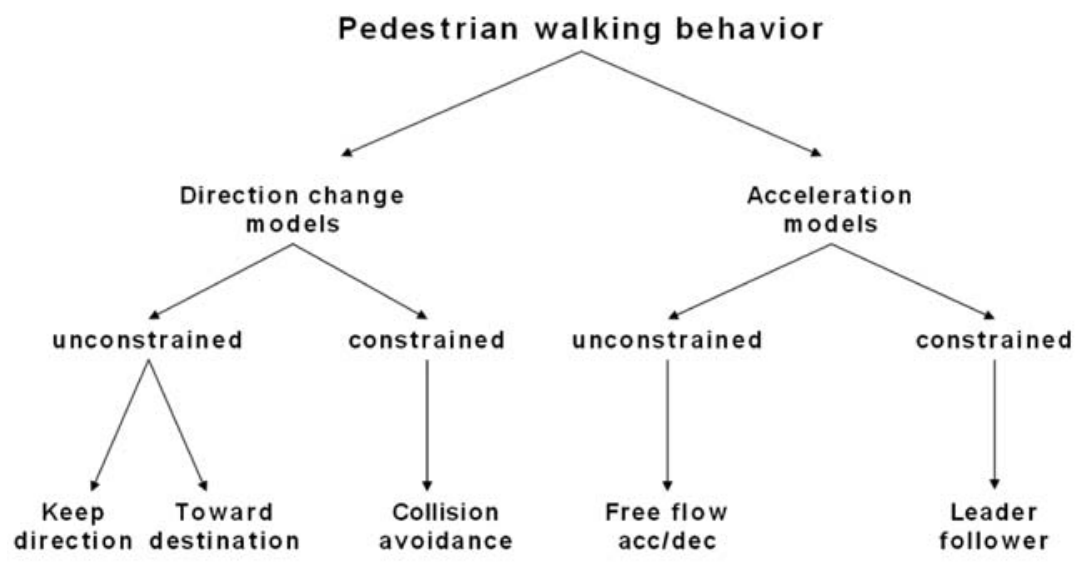

Figure 2: Conceptual framework for pedestrian walking behavior.

» toward destination We assume the final destination of an individual as an exogenous variable in the model, being fixed at the strategical decision level. Being pedestrian movements $2 \mathrm{D}$ displacements, we assume that both the difference in direction and the distance from the final destination influence individuals. With the toward destination behavior we identify the tendency of pedestrians to move, when is possible, directly toward their final destinations.

These first two behaviors can be interpreted and located in a more general context. First of all, they are unconstrained models for direction changes, i.e. they do not depend on the presence of other individuals. Referring to the hierarchical decision process described above, individuals choose their final destinations at a strategic level while a route choice and/or intermediate destination choice happen at the tactical level. Such higher level choices are reflected naturally on the short term behavior, captured at the operational level by the keep direction and toward destination behavioral patterns.

॥ free flow acceleration/decelerationWe refer here to the free flow, unconstrained, acceleration behavior. The main assumption is that the acctractiveness for an acceleration (deceleration) depends on the current individual speed module. Indeed, someone who is already walking fast has less incentive for further accelerations with respect to someone who is walking slowly. This model for accelerations is conceptually related to the idea of a maximum physical speed. Such a value is unobserved and individual dependent, being related to socio-economics characteristics such as age, gender, weight etc. ... In our model we use the maximum observed speed module in the data and for simplicity we assume it as homogeneous over the population

» leader follower A common phenomenon observed in highly crowded situations is the lane formation. People behave identifying leaders in their surrounding 
environment and choose to follow them as an optimal solution to better attain their goals. Individuals satisfying at the leader conditions represent the potential leaders. We allow for more potential leaders for each radial direction. A leader choice process is assumed, leading to one leader for each direction, constraining the acceleration (deceleration) behavior of the decision maker

collision avoidance Individuals change direction in order to avoid possible collisions with other individuals. As for the leader follower, potential colliders are defined on the base of certain collider conditions. An implicit collider choice process is assumed, leading to one collider for each direction, influencing the direction change behavior of the decision maker

\section{The pedestrian behavior model}

Three speed regimes and 11 radial directions give rise to a choice set composed by 33 alternatives, each of them represented by its central point.

\section{Systematic utilities}

The systematic utilities of the full model are reported in equation 1:

\begin{tabular}{|c|c|}
\hline \multicolumn{2}{|l|}{$\mathrm{V}_{\mathrm{vdn}}=\beta_{d i r} d i r_{d n}$} \\
\hline$\beta_{\mathrm{acc}} \mathrm{I}_{\mathrm{v}, \mathrm{acc}}\left(\nu_{\mathrm{n}} / \nu_{\max }\right)^{\lambda_{a c c}}$ & + \\
\hline$\beta_{d e c} I_{v, d e c}\left(v_{n} / v_{\max }\right)^{\lambda_{d e c}}$ & + \\
\hline$\beta_{\text {ddest }} d d e s t_{v d n}$ & + \\
\hline$\beta_{d d i r} d d i r_{d n}$ & + \\
\hline$I_{v, a c c} I_{a c c}^{L} \alpha_{a c c}^{L} D_{L_{L}}^{\rho_{a c c}^{L}} \Delta v_{L_{L}}^{\gamma_{a c c}^{L}} \Delta \theta_{L_{L}}^{\delta_{a c c}^{L}}$ & + \\
\hline$I_{v, \text { dec }} I_{\text {dec }}^{L} \alpha_{d e c}^{L} D_{L_{L}}^{\rho_{\text {dec }}^{L}} \Delta v_{L_{L}}^{\gamma_{\text {dec }}^{L}} \Delta \theta_{L_{L}}^{\delta_{d e c}^{L}}$ & + \\
\hline$I_{d, d_{n}} I_{C} \alpha_{C} e^{-\rho_{C} D_{C}} \Delta v_{C}^{\gamma_{C}} \Delta \theta_{C}^{\delta_{C}}$ & \\
\hline
\end{tabular}

where all the $\beta$ parameters as well as $\lambda_{\text {acc }}, \lambda_{\text {dec }}, \alpha_{\text {acc }}^{\mathrm{L}}, \rho_{\text {acc }}^{\mathrm{L}}, \gamma_{\text {acc }}^{\mathrm{L}}, \delta_{\text {acc }}^{\mathrm{L}}, \alpha_{\mathrm{dec}}^{\mathrm{L}}, \rho_{\text {dec }}^{\mathrm{L}}, \gamma_{\text {dec }}^{\mathrm{L}}, \delta_{\text {dec }}^{\mathrm{L}}, \alpha_{\mathrm{C}}, \rho_{\mathrm{C}}$, $\gamma_{\mathrm{C}}, \delta_{\mathrm{C}}$ are unknown and have to be estimated. Note that this specification is the result of an intensive modeling process, where many different specifications have been tested. For the keep direction and free flow acceleration behaviors (the first 3 terms in equation 1) we keep the same specification as defined in [1]. For the toward destination behavior (the 4th and 5th terms in 1) the distance of an alternative from the destination has been added (the $\beta$ ddestddestvdn term), with no conceptual changes with respect to its previous specification. In the following, the new elements introduced in this work are described. 
》

\section{Leader follower}

The leader follower behavior has been inspired by car following models, used in transport engineering since the early work of [4]. In such models, a subject car follows a leader one, reacting to its actions under different criteria. Here we adapt this idea to the case of pedestrians, using a sensitivity-stimulus framework (see [2]).

The leader follower behavior is modeled as a constrained acceleration (deceleration) term. The indicator function $I_{\text {vacc }}$ in equation 1 is 1 if $\mathrm{v}=\mathrm{v}_{\text {ace }}$, that is, if the alternative corresponds to an acceleration and 0 otherwise. $I_{v, d e c}$ is similarly defined. We consider several potential leaders in the surrounding of the current decision maker (see Figure 3(b)). An individual $k$ is defined as a potential leader if she satisfies at the leader conditions. We can informally define potential leaders as those individuals inside the region of interest, not so far from the decision maker and having movement directions not so different from the radial cone where they lies. We formalise such ideas by means of the indicator function $I_{\mathrm{g}}^{\mathrm{k}}$ :

$I_{g}^{k}=\left\{\begin{array}{l}1, \text { if } d_{l} \leq d_{k} \leq d_{r} \\ \text { if } 0<D_{k} \leq D_{t h} \\ \text { if } 0<\left|\Delta \theta_{k}\right| \leq \Delta \theta_{t h} \\ 0, \text { otherwise. }\end{array}\right.$

where $d_{l}$ and $d_{\mathrm{r}}$ represent the bounding left and right directions of the choice set defining the region of interest (see Figure 3(b)) while $d_{\mathrm{k}}$ is the direction identifying the pedestrian $k$ position. $D_{\mathrm{k}}$ is the distance between pedestrian $k$ and the decision maker, $\Delta \theta_{k}=\theta_{k}-\theta_{\mathrm{d}}$ is the difference between the movement direction of pedestrian $k\left(\theta_{k}\right)$ and the angle characterizing direction $d$, i.e. the direction identifying the radial cone where individual $k$ lies $\left(\theta_{d}\right)$. The two thresholds $D_{\text {th }}$ and $\Delta \theta_{\text {th }}$ are fixed at the values $D_{\text {th }}=5 D_{\text {max }}$, where $D_{\max }$ is the radius of the choice set, and $\Delta \theta_{\mathrm{th}}=10$. We assume an implicit leader choice process executed by the decision maker herself and modelled choosing as leader for direction $d$ that pedestrian satisfying at the leader conditions, and which is at distance $D_{\mathrm{L}}=\min _{\mathrm{k \in K}}\left(D_{\mathrm{k}}\right)$, as illustrated if Figure 3(b), where $K$ is the number of potential leaders in the radial cone characterized by direction $d$. The corresponding indicator function is $I_{\mathrm{g}}^{\mathrm{L}}$, where $\mathrm{g}=\{\mathrm{acc}, \mathrm{dec}\}$ indicates accelerations and decelerations, based on the sign of the leader's relative speed $\Delta v_{\mathrm{L}}$ defined as:

$\Delta v_{L}=\left|v_{L}-v_{n}\right|$ 
where $v_{\mathrm{L}}$ and $v_{\mathrm{n}}$ are the leader's speed module and the decision maker's speed module, respectively. The acceleration of the decision maker as a response to the presence of the leader individual is given by:

acceleration $=$ sensitivity $\times$ stimulus

The sensitivity term of the model is a non linear function of the leader distance $D_{\mathrm{L}}$, defined as follows:

sensitivity $=f\left(D_{L}\right)=\alpha_{g}^{L} D_{L}^{\rho_{g}^{L}}$

with $D_{\mathrm{L}}$ defined above. The parameters $\alpha_{g}^{L}$ and $\rho_{g}^{L}$ have to be estimated. The decision maker reacts to stimuli coming from the chosen leader. We model the stimulus as a function of the leader's relative speed $\Delta v_{\mathrm{L}}$ and the leader's relative direction $\Delta \theta_{\mathrm{L}}$ as follows:

stimulus $=f\left(\Delta v_{L}, \Delta \theta_{L}\right)=\Delta v_{L}^{\gamma_{g}^{L}} \Delta \theta_{L}^{\delta_{g}^{L}}$

where the parameters $\gamma_{\mathrm{g}}^{\mathrm{L}}$ and $\delta_{\mathrm{g}}^{\mathrm{L}}$ have to be estimated. A leader acceleration (deceleration) induces a decision maker acceleration (deceleration). A substantially different movement direction in the leader reduces the influence of the latter on the decision maker herself. The use for such functional forms finds its justification in the numerous previous works on car following models.

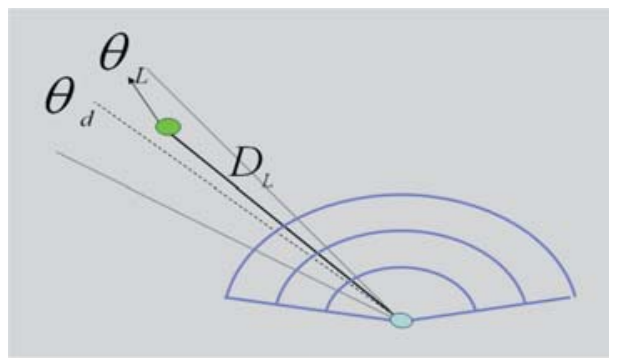

(a)

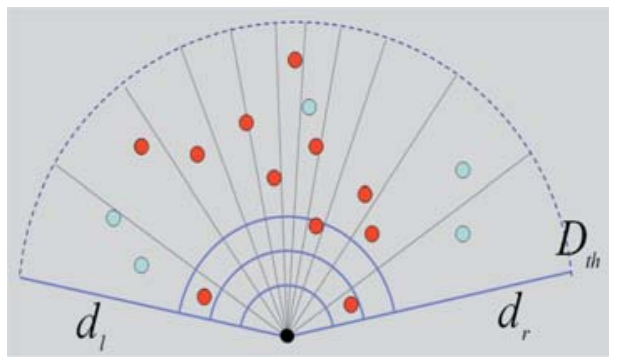

(b)

Figure 3: The elements defining the leaders. More than one potential leader is allowed for each radial cone. That one at the minimum distance from the decision maker is chosen as leader 
》

\section{Collision avoidance}

Based on the dynamic characteristics of other individuals the decision maker changes her own direction, attempting to avoid collisions with other pedestrians. We keep for this model a sensitivity/stimuls formulation. Following the approach used for the leader follower model, we assume different potential colliders for each of the 11 directions. Using a similar notation as before, a pedestrian $k$ represents a potential collider if she satisfies at the collider condictions, as defined by the following indicator function:

$I_{C}^{k}=\left\{\begin{array}{l}1, \text { if } d_{l} \leq d_{k} \leq d_{r} \\ \text { if } 0<D_{k} \leq D_{t h}^{\prime} \\ \text { if } \frac{\pi}{2} \leq\left|\Delta \theta_{k}\right| \leq \pi \\ 0, \text { otherwise. }\end{array}\right.$

where $d_{1}, d_{\mathrm{r}}$ and $d_{\mathrm{k}}$ are the same as those defined for the leader follower model. $D_{\mathrm{k}}$ is now the distance between individual $\mathrm{k}$ and the center of the alternative, $\Delta \theta_{\mathrm{k}}=\theta_{k}-\theta_{\mathrm{dn}}$ is the difference between the movement direction of pedestrian $k$, $\theta_{\mathrm{k}}$, and the movement direction of the decision maker, $\theta_{\mathrm{dn}}$. The value of the distance threshold is now fixed to $D_{\mathrm{th}}=10 d_{\max }$. We use a larger value for such a threshold compared to the leader follower model, assuming the collision avoidance behavior being a longer range interaction, happening also at a lower density level. We assume an implicit collider choice process, executed by the decision maker and modeled choosing as collider for direction $d$ that potential collider having $\Delta \theta_{\mathrm{C}}=\max _{k \in K}\left|\Delta \theta_{\mathrm{k}}\right|$, where $K$ is the number of potential colliders in the radial cone characterized by direction $d$. The related indicator function is $I_{\mathrm{C}}$. The scenario for the potential colliders is similar to the leader follower model and is reported in figures 4(a) and 4(b). We assume a sensitivity function being a function of the distance $D_{\mathrm{C}}$ between the collider position and the center of the alternative, definded as follows:

sensitivity $=f\left(D_{C}\right)=\alpha_{C} e^{\rho_{C} D_{C}}$

where the parameters $\alpha_{\mathrm{C}}$ and $\rho_{\mathrm{C}}$ have to be estimated. We choose the exponential form to keep the same functional form as that used in [1]. The decision maker reacts to stimuli coming from the collider. We model the stimulus as a function of two variables:

stimulus $=f\left(\Delta v_{C}, \Delta \theta_{C}\right)=\Delta v_{C}^{\gamma_{c}} \Delta \theta_{C}^{\delta_{C}}$ 
with $\Delta \theta_{\mathrm{C}}$ defined above and $\Delta v_{\mathrm{C}}$ defined as follows:

$$
\Delta v_{C}=\left|v_{C}\right|+\left|v_{n}\right|
$$

with $v_{\mathrm{C}}$ and vn the collider and decision maker speed modules respectively. The parameters $\gamma_{\mathrm{C}}$ and $\delta_{\mathrm{C}}$ have to be estimated. Individuals walking against the decision maker at higher speeds and in more frontal directions generate stronger reactions, weighted by the sensitivity function.

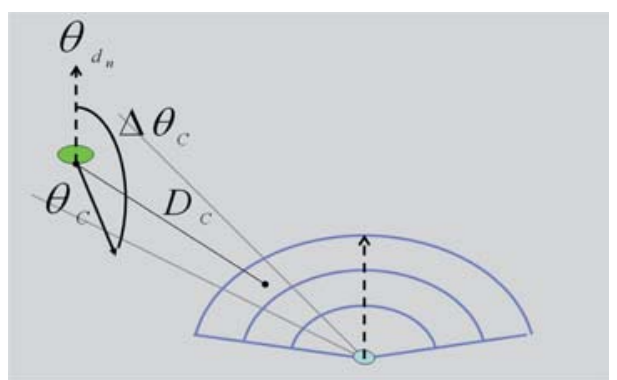

(a)

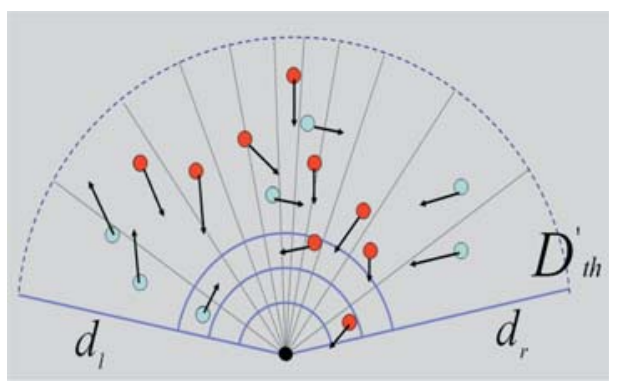

(b)

Figure 4: The elements defining the colliders. More than one potential collider is allowed for each radial cone. That one forming the maximum angle DeltaThet ${ }_{\mathrm{C}}$ is chosen as collider

\section{The random term}

We keep the same cross nested logit specification used in our previous work. Such a model allows for flexible correlation structures in the choice set, keeping a closed form solution. The probability of choosing alternative $i$ within the choice set $C$ for a given choice maker is:

$$
P(i \mid C)=\frac{\Sigma_{m}\left(\alpha_{i m} y_{i}\right)^{\mu_{m}}\left(\Sigma_{j}\left(\alpha_{j m} y_{j}\right)^{\mu_{m}}\right)^{\frac{\mu}{\mu_{m}}-1}}{\Sigma_{m}\left(\Sigma_{j \in C}\left(\alpha_{j m} y_{j}\right)^{\mu_{m}}\right)^{\frac{\mu}{\mu_{m}}}}
$$

where $\alpha_{\text {jm }} \geq 0 \forall j, m ; \mu>0 ; \mu_{\mathrm{m}}>0 \forall m ; \mu \leq \mu_{\mathrm{m}} \forall m$. The systematic utility function enters this formula as $y_{\mathrm{i}}=e^{\mathrm{Vi}}$. We assume a correlation structure depending on the speed and direction and we identify five nests: accelerated, constant speed, decelerated, central direction and not central direction. Given the lack of any a priori information, we fix the degrees of membership to the different nests $\left(\alpha_{j m}\right)$ to the constant value 0.5 (see [5]). 


\section{Data}

The datasets used to estimate the model consists of pedestrian trajectories manually tracked from video sequences. We have pooled together two different datasets, collected separately in Switzerland and Japan.

The swiss dataset This part of the dataset consists of 36 pedestrian trajectories, manually tracked from a digital video sequence. The scene has been recorded out of the Lausanne metro station in 2002. For a detailed description of this dataset we refer the reader to [1].

The japanese dataset This dataset has been collected in Sendai, Japan, on August 2000 (see [6]). The video sequence has been recorded from the 6th floor of the JTB parking building (around 19 meter height), situated at a large pedestrian crossing point. Two main pedestrian flows cross the street, giving rise to a large number of interactions. In this context, 190 pedestrian trajectories have been manually tracked, with a time step of 1 second. The collected data contain the pedestrian identifier, the time step and the image coordinates. The mapping between the image plane and the walking plane is approximated by a 2D-affine transformation, whose parameters are learnt by linear regression.

\section{Results}

Table 1 reports the estimation results for the CNL specification. We have estimated the unknown parameters of the model using the Biogeme package ([7]). It is a freeware package for the estimation of a wide range of random utility models.

The negative signs for the $\beta_{\text {ddir }}$ and $\beta_{\text {ddist }}$ indicate that a cost is perceived for those alternatives farther from the final destination, both in distance and direction. The $\beta_{\text {dir }}$ estimated value reflects the tendency of individuals to avoid frequent direction changes. People decide their final destinations at a strategic level, their routes and paths at a tactical level ([8]). These decisions are reflected on a short time horizon and are coherent with negative signs for the $\beta_{\text {ddir }} \beta_{\text {ddist }}$ and $\beta_{\text {dir }}$ coefficients. This conclusion is also accordant with other previous studies on the idea that individuals move through spaces along paths that minimize the angular displacements (see [9] for more details). $\beta_{\text {acc }}$ and $\beta_{\text {dec }}$ signs confirm the idea that changes in speed reduce the utility of the corresponding alternatives, indicating a preference of pedestrians for constant speed values. A positive sign for the elasticity parameter $\lambda_{\text {acc }}$ reflects the fact that the attractiveness for accelerations is a non linear function of the current speed value, as already shown in [1]. The elasticity for decelerations, $\lambda_{\text {dec }}$, has not been estimated significantly different from zero. This first part of the estimated parameters correspond to the unconstrained behaviors, depending only on the decision maker movements.

The $\alpha_{\text {ace }}^{\mathrm{L}}, \rho_{\text {acc }}^{\mathrm{L}}, \gamma_{\text {acc }}^{\mathrm{L}}$ and $\delta_{\text {acc }}^{\mathrm{L}}$ describe the leader follower behavior in case of an accelerated leader. The presence of such an accelerated leader increases the utility of the accelerated 
alternatives $\left(\alpha_{\text {acc }}^{\mathrm{L}}>0\right)$. The negative sign for $\rho_{\text {acc }}^{\mathrm{L}}$ reflects the fact that the distance of the leader is negatively correlated with the induced acceleration, as expected. Increments in the absolute value of the leader relative speed induce greater accelerations, as indicated by $\gamma_{\text {acc }}^{\mathrm{L}}>0$. The leader relative direction coefficient, $\delta_{\text {acc }}^{\mathrm{L}}$, has not been estimated signifi-

\begin{tabular}{|c|c|c|c|}
\hline $\begin{array}{c}\text { Variable } \\
\text { name }\end{array}$ & $\begin{array}{c}\text { Coefficient } \\
\text { estimate }\end{array}$ & $t$ test 0 & $t$ test 1 \\
\hline$\beta_{\text {ddir }}$ & -0.061 & -19.066 & \\
\hline$\beta_{\text {ddist }}$ & -1.614 & -1.9749 & \\
\hline$\beta_{\mathrm{dir}}$ & -0.027 & -11.342 & \\
\hline$\beta_{\text {acc }}$ & -19.822 & -5.847 & \\
\hline$\beta_{\mathrm{dec}}$ & -2.069 & -2.651 & \\
\hline$\lambda_{\text {acc }}$ & 0.969 & 26.880 & \\
\hline$\alpha_{\mathrm{acc}}^{\mathrm{L}}$ & 4.883 & 3.368 & \\
\hline$\rho_{\mathrm{acc}}^{\mathrm{L}}$ & -0.657 & -3.034 & \\
\hline$\gamma_{\mathrm{acc}}^{\mathrm{L}}$ & 0.869 & 9.877 & \\
\hline$\alpha_{\mathrm{dec}}^{\mathrm{L}}$ & 4.061 & 6.278 & \\
\hline$\rho_{\mathrm{dec}}^{\mathrm{L}}$ & -0.481 & -4.280 & \\
\hline$\gamma_{\mathrm{dec}}^{\mathrm{L}}$ & 0.524 & 9.089 & \\
\hline$\delta_{\mathrm{dec}}^{\mathrm{L}}$ & -0.892 & -1.642 & \\
\hline$\alpha_{\mathrm{C}}$ & -0.0058 & -4.639 & \\
\hline$\rho_{\mathrm{C}}$ & -0.313 & 6.748 & \\
\hline$\gamma_{\mathrm{C}}$ & 0.781 & 3.318 & \\
\hline$\mu_{\text {const }}$ & 1.597 & 32.413 & 12.119 \\
\hline$\mu_{\text {not_central }}$ & 1.487 & 15.765 & 5.160 \\
\hline$\mu_{2}$ & 0.591 & - & -8.565 \\
\hline \multicolumn{4}{|c|}{ Sample size $=10783$} \\
\hline \multicolumn{4}{|c|}{ Number of estimated parameters $=19$} \\
\hline \multicolumn{4}{|c|}{ Init log-likelihood $=-78558.3$} \\
\hline \multicolumn{4}{|c|}{ Final log-likelihood $=-22572.7$} \\
\hline \multicolumn{4}{|c|}{ Likelihood ratio test $=30260.3$} \\
\hline $\bar{\rho} 2=0.4007$ & & & \\
\hline
\end{tabular}

Table 1: CNL estimation results 
cantly different from 0 . Similar interpretation for the decelerated leader parameters $\alpha_{\text {dec }}^{\mathrm{L}}$, $\rho_{\text {dec }}^{\mathrm{L}} \gamma_{\text {dec }}^{\mathrm{L}}$ and $\delta_{\text {dec }}^{\mathrm{L}}$. We keep $\delta_{\text {dec }}^{\mathrm{L}}$ in the model, even if it is not significant (t-test =1.642). Its negative sign might be explained as a lower influence of those leaders having movement directions quite different from the radial cones where they are, as expected. The $\alpha_{C}, \rho_{C}, \gamma_{C}$ and $\delta_{C}$ describe the constrained direction change behavior, i.e. collision avoidance. The negative sign for $\alpha_{C}$ indicates a lower utility for those directions where there is a collider, as expected. Colliders farther from the alternatives have a lower negative influence on the utilities $\left(\rho_{\mathrm{C}}<0\right)$. Higher values for the collider relative speed, indicating early possible collisions, decrease the utility values $\left(\gamma_{C}>0\right)$. The value of $\delta_{C}$ is not estimated. It has been fixed arbitrarily to 1 .

Two over five nest coefficients have been estimated significantly different from 1. $\mu_{\text {const }}$ and $\mu_{\text {not_central }}$ capture the correlation between the alternatives in the constant speed and the not central direction nests, respectively. Finally, two different scale parameters have been used to capture the heterogeneity in the two different datasets. We have fixed to 1 the scale related to the Japanese data and the scale related to the Swiss sample $(\mu 2)$ has been estimated. Its value is significantly different from 1 , as reported in Table 1 .

\section{Summary}

We have presented here a development of our previous work on pedestrian modeling. A discrete choice framework is used, interpreting the walking process as a sequence of choices, step by step. In this paper we have generalized the approach, identifying direction changes and accelerations behaviors for a short time range pedestrian model. Both such behaviors have unconstrained and constrained formulations. The first refer to decisions taken by individuals based on their kinematic state and on decisions taken at a strategical and/or tactical levels (destination choice, route choice). The seconds refer to constrained accelerations (decelerations) induced by individuals perceived as leaders, who are defined on the base of certain leader conditions. Similarly, constrained direction change behaviors are described in terms of collision avoidance patterns, induced by the presence of colliders, defined on the base of certain collider conditions. The model shows a good explanatory power with respect to the datasets used for estimation. The heterogeneity in the data has been captured by two different scale parameters. Correlations between alternatives are captured by means of a CNL formulation.

Interesting extensions to our approach would include the use of controlled experimental conditions, allowing for the use of socio-economic variables, such as age, sex, weight etc.... The influence of the spatial layout should also be included, being the model a pure human-to-human interaction model. «

\section{Acknowledgments}

This work is supported by the Swiss National Science Fundation under the NCCRIM2 project.We are grateful to Kardi Teknomo for the NTXY dataset. 


\section{References}

1. G. Antonini, M. Bierlaire, and M. Weber. Discrete choice models of pedestrian behavior, 2004. Under review for publication on Transportation Research.

2. T.Toledo. Integrated Driving Behavior Modeling. PhD thesis, Massachusetts Institute of Technology, 2003.

3. K.I.Ahmed. Modeling drivers' acceleration and lane changing behaviors. $\mathrm{PhD}$ thesis, Massachusetts Institute of Technology, 1999.

4. R. Reuschel. Fahrzeugbewegungen in der kolonne. Osterreichisches Ingenieur Archiv, 4:193-215, 1950.

5. Michel Bierlaire. A theoretical analysis of the cross-nested logit model. Accepted for publication in Annals of Operations Researchs.

6. K. Teknomo, Y. Takeyama, and H. Inamura. Review on microscopic pedestrian simulation model. In Proceedings Japan Society of Civil Engineering Conference, 2000 .

7. Michel Bierlaire. An introduction to BIOGEME Version 0.6, February 2003. roso. epfl.ch/biogeme.

8. S.P. Hoogendoorn, P.H.L. Bovy, and W.Daamen. Microscopic pedestrian wayfinding and dynamics modelling. In M. Schreckenberg and S.D. Sharma, editors, Pedestrian and Evacuation Dynamics, pages 123-155. Springer, 2002.

9. A. Turner. Angular analysis. In In Proceedings 3rd International Symposium on Space Syntax, pages 30.1-30.11, 2001. 